\title{
AN AFM PROBE CONTROLLER DESIGN BASED ON $\mu$-SYNTHESIS
}

Kuo-Jung Lan, Jia-Yush Yen, Chih-Kung Lee, Yi-Ming Lin, and Cheng-Hung Chen

Asian Journal of Control

Vol. 7, No.1, pp. 12-19, March 2005 


\title{
AN AFM PROBE CONTROLLER DESIGN BASED ON $\mu$-SYNTHESIS
}

\author{
Kuo-Jung Lan, Jia-Yush Yen, Chih-Kung Lee, Yi-Ming Lin, and Cheng-Hung Chen
}

\begin{abstract}
The atomic force microscope (AFM) is one of the most important tools for measuring atomic resolution. The AFM system maintains constant force between a tip and the sample in order to track the sample topography. The controller that maintains the constant interaction force plays a significant role in measurement accuracy. This paper presents a $\mu$-synthesis controller design to deal with model uncertainty and establish a measurement error bound. The system's nonlinearity and the set-point drift are lumped into a multiplicative uncertainty. The performance bound allows specification of the error magnitude over the frequency range. Simulation results show that the proposed control can tolerate uncertainties. The error spectrum from the experiments shows consistency with the design specifications. Images were taken to compare $\mu$-synthesis control with a well-tuned PID control at a $480 \mu \mathrm{m} / \mathrm{s}$ scan rate. The results verify the outstanding performance of the $\mu$ controller.
\end{abstract}

KeyWords: Atomic force microscope, multiplicative uncertainty, $\mu$-synthesis, and PID control.

\section{INTRODUCTION}

The scanning based atomic force microscope (AFM) measures objects with atomic resolution. It is finding increased use in the inspection of IC chips, computer hard disks, optical disks, etc. As feature sizes grows smaller in these applications [1], the need for greater accuracy and faster scanning speed becomes greater.

Often, the resolution of an AFM depends on the control system that maintains the interaction force between the probe tip and sample. An AFM uses a laser light lever bounced off the scanning probe tip to measure small deflections. A piezoelectric actuator moves the probe to maintain a constant interaction force. The performance of the controller in controlling the movement of the scanning tip plays a key role in measurement accuracy. The ability to track the surface profile to high accuracy determines the steady-state resolution. The ability of the tip to follow rapid profile changes determines the scanning speed of

Manuscript received March 5, 2004; accepted April 8, 2004.

Kuo-Jung Lan, Jia-Yush Yen, Yi-Ming Lin, and ChengHung Chen are with Department of Mechanical Engineering, National Taiwan University, Taipei, Taiwan 10617, R.O.C..

Chih-Kung Lee is with Institute of Applied Mechanics, National Taiwan University, Taipei, Taiwan 10617, R.O.C. the AFM. High performance control for such a probe system is, thus, necessary. It is further desirable that the control error be specified because the control error directly affects the measurement result. No limitation is imposed by the piezoelectric actuator. Therefore, the bound on the control error is directly translated into the measurement resolution.

Previous research works have focused on both the modeling and control aspects of the probe control problem. Schitter et al. used the $H_{\infty}$ design to develop a fast scanning AFM probe controller that can scan five times faster then the classical systems [2]. Battistom et al. used the fuzzy logic controller in a combined AFM/STM [3]. Because of the nonlinearity associated with the AFM system, some researchers have tried to use the nonlinear control technique to achieve asymptotic tracking [4]. The authors of [5] and [6] have developed models for scanners, the cantilevers, and tip-sample interaction. Osamash in [7] showed that the dynamics of AFM are dependent on the sample material, the contact force set-point, and the amplitude of the disturbance. The issue of measurement tolerance has not been addressed.

This paper presents a $\mu$-synthesis controller for the AFM probe control system. The $\mu$-synthesis setup allows specification of the force tolerance; thus, it is possible to quantify the amount of error introduced by the control 
action. The force-distance relationship can then be used to establish frequency bounds for the measurement error. Nonlinear AFM dynamic effects, such as hysteresis, creeping, tip-sample interaction, cantilever nonlinearity, and set-point effects, are dealt with by introducing an adequate multiplicative uncertainty into the design specification. The controller design is, thus, robust. Because the low frequency error bound is properly designed, the resultant controller guarantees a control error of less than one percent in the low frequency region. Simulation results show that the control is tolerant to uncertainties, and experimental results confirm the error bound. Scanned images demonstrate that the proposed control delivers better quality pictures than a classical PID controller at a high scan rate.

In this paper, we present our approach to system identification using curve-fitting methods for the overall system. The proposed system includes a position-sensitive photo-detector (PSPD), piezo scanner, tip-sample interaction, and cantilever dynamics.

This paper is divided into seven major sections. Section 2 describes the AFM system. Section 3 describes the mathematical model for tip-sample interaction and the system identification procedure. Sections 4 and 5 discuss the controller design and the performance based on computer simulations. Section 6 presents the implementation and experimental results, and Section 7 presents conclusions.

\section{SYSTEM DESCRIPTION}

The AFM uses a sharp tip to scan the surface of a sample. The tip located at the end of a $450 \mu \mathrm{m}$ flexible cantilever is a few micrometers long, and its radius is smaller than $10 \mathrm{~nm}$. When the tip approaches the sample, the interaction causes the cantilever to bend around the peaks and valleys of the sample surface. A laser beam bounced off the end of the cantilever onto a photo detector measures the amount of deflection. Figure 1 shows the system configuration.

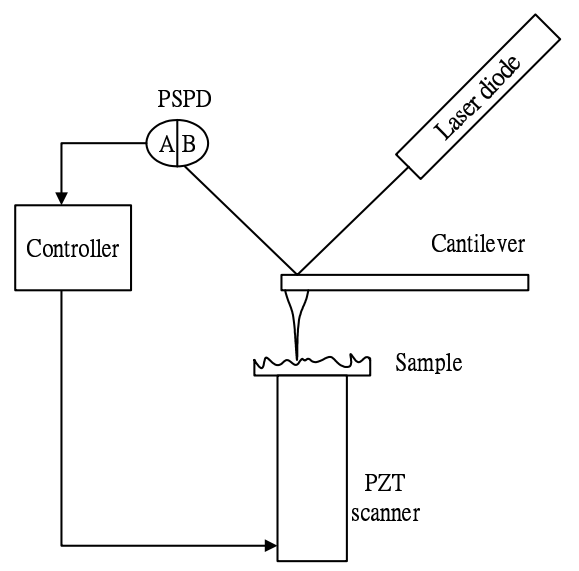

Fig. 1. The AFM system configuration
The piezo tube with a $100 \mu \mathrm{m}$ range provides the three-dimensional motion of the sample. There is a constant relationship between the Van der Waals force and the distance from the probe. The controller senses the bending of the cantilever and moves the scanner stage to maintain constant force. In the so-called "contact region," the cantilever is held less than a nanometer from the sample surface, and the interaction between the cantilever and the sample is repulsive; in the "non-contact region," the cantilever is held a few nanometers from the sample surface, and the interaction is attractive. In this study, the AFM was operated in the contact mode, also known as the repulsive mode. The AutoProbe LS (as shown in Fig. 2) from Park Scientific Instruments was employed in this study. It was modified to operate with the controller developed at NTU. C++ code was written to execute the control and data storage. The host PC was Pentium-based with an Advantech I/O card. Data analysis was performed with the MATLAB software.



Fig. 2. The AutoProbe LS AFM

\section{SYSTEM MODELING AND IDENTIFICATION}

\subsection{Tip-sample model in the contact region}

Consider a Dugdale model with adhesion forces; the model can be expressed by the following equations ([5] and [8]). The tip deflection of the AFM probe is expressed as

$$
\begin{aligned}
\delta=\frac{a^{2}}{R} & -\frac{4 a \lambda}{3}\left(\frac{4 E}{3 \pi w R^{2}}\right)^{1 / 3} \sqrt{m^{2}-1}, \\
F(\delta)= & a^{3} \frac{4 E}{3 R} \\
& -\lambda a^{2}\left(\frac{4 E}{3 \pi w R^{2}}\right)^{2 / 3}\left[\sqrt{m^{2}-1}+m^{2} \sec ^{-1}(m)\right],
\end{aligned}
$$




$$
\begin{array}{r}
3 \lambda a^{2}\left(\frac{4 E}{3 \pi w R^{2}}\right)^{2 / 3}\left[\sqrt{m^{2}-1}+\left(m^{2}-2\right) \sec ^{-1}(m)\right] \\
+8 \lambda^{2} a\left[-m+1+\sqrt{m^{2}-1} \sec ^{-1}(m)\right]=6
\end{array}
$$

where $\delta$ is the tip sample relative displacement, $R$ is the radius of the tip and sample, $E$ is the combined elastic modulus, $F(\cdot)$ is the contact force, $w$ is the adhesion constant, $\sigma_{0}$ is the Dugdale attractive force, and $\lambda=$ $\sigma_{0}\left(\frac{9 R}{2 \pi w E^{2}}\right)^{1 / 3}$. The variables $a$ and $m$ are dummy variables to be determined.

The cantilever equation of motion can be expressed as a mass-spring-damper system:

$$
\ddot{x}(t)+2 \xi \omega \cdot \dot{x}(t)+\omega^{2} x(t)=\frac{1}{m} F\left(x(t)-u(t)+x_{0}\right),
$$

where $m$ is the reduced mass of the cantilever, $x_{0}$ is the static-constant distance between the tip and sample, $x$ is the displacement of the tip, and $u$ is the topography of the sample. The cantilever is $1.7 \mu \mathrm{m}$ thick and $443 \mu \mathrm{m}$ long. Its nominal force constant is $0.11 \mathrm{~N} / \mathrm{m}$, and its resonance frequency is $11 \mathrm{kHz}$. The reduced mass is $23 \mathrm{ng}$. A typical tip radius is smaller than $10 \mathrm{~nm}$. By linearizing the interaction force in Eq. (4), one can get a linear system representation of the AFM control system.

\subsection{System identification}

To obtain an accurate model for the control system, an HP3563A dynamic frequency analyzer was used to measure the frequency response. The input to the system was the piezo actuator voltage, and the output was the PSPD signal. The force setpoint in this case was set to $20 \mathrm{nN}$. Figure 3 shows the experimental frequency response and the curve fitted system model. The first resonance of the system occured at $850 \mathrm{~Hz}$, which is at a bending mode of the cantilever.


Fig. 3. Frequency response comparison
The identified system is a third order one with one stable real pole at $140 \mathrm{~Hz}$ and a complex pole at $850 \mathrm{~Hz}$. The unmatched portion in the high frequency region will be treated as the unmodeled dynamics. It should be noted that the frequency response of the plant may change with different set-points; therefore, uncertainty treatment is necessary.

\section{IV. $\mu$-SYNTHESIS CONTROL DESIGN}

\subsection{Multiplicative uncertain model}

The AFM system response change depends on the force set-point. A multiplicative uncertainty is used to adequately describe the frequency-dependent percentage uncertainty ([9] and [11]):

$$
\tilde{G}=G\left(I+W_{u} \Delta\right),
$$

where $\tilde{G}$ is the plan with uncertainties. We assume that the nominal plan $G$ has the same numbers of poles in the right half of the plane. At each frequency, $\left|W_{u}(j \omega)\right|$ represents the maximum error percentage between $G$ and $\tilde{G}$, and $\Delta$ is a transfer function that satisfies $\max _{\omega \in R}|\Delta(j \omega)| \leq 1$. The system is then put into the Linear Fractional Transformation (LFT) form for the $\mu$-synthesis process.

\subsection{Choosing performance criterion}

For system $G$ with compensator $K$, the closed-loop performance can be specified by the sensitivity transfer function matrix $S=(I+G K)^{-1}$ :

$$
S=(I+P C)^{-1} .
$$

By augmenting an artificial signal filter, $W_{p}$, at the error output, the $\mu$-synthesis setup guarantees a controller that achieves $\left\|W_{p} S\right\|_{\infty}<1, \forall \omega$. The augmented signal filter $\left|W_{p}\right|$ now serves as a performance criterion for the sensitivity transfer function.

\subsection{Using $\mu$ to analyze robust stability}

Consider the standard block diagram with a controller $K$ shown in Fig. 4 [10].



Fig. 4. The system setup with uncertainty $\Delta$ 
$\Delta$ in Fig. 4 represents a structured uncertainty with diagonal terms satisfying $\max _{\omega \in R}\left|\Delta_{i}(j \omega)\right| \leq 1$. A block diagram of the uncertain closed-loop system illustrating the robustness criteria and the performance objective (from $r$ $\rightarrow e$ ) is shown in Fig. 5. The variables $r, u, y, d$, and $e$ are the force set-point, control effort, PSPD signal, sample topography, and force error, respectively. The blocks $G$, $W_{u}, W_{p}$, and $K$ are the nominal plant, multiplicative input uncertainty model, error performance upper bound, and controller to be determined, respectively.

The choices for $W_{p}$ and $W_{u}$ are shown in Fig. 6. The design uses an error bound of $-40 \mathrm{~dB}$ for signals below $1 \mathrm{~Hz}$. Because the model above $1 \mathrm{kHz}$ is uncertain, we allow for $W_{u}$ greater than $0 \mathrm{~dB}$ in the high frequency region. The $W_{u}$ function is determined by the uncertain region of the open loop frequency response, which is measured under certain conditions, including different set-points of force, different tips, and different bending of the piezo tube.

\section{SIMULATION}

\subsection{Simulation}

The $\mu$-synthesis procedure resulted in a $7^{\text {th }}$ order controller. For more efficient controller implementation,

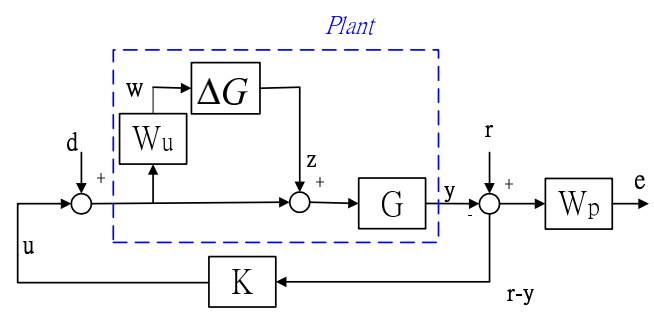

Fig. 5. Uncertain closed loop system

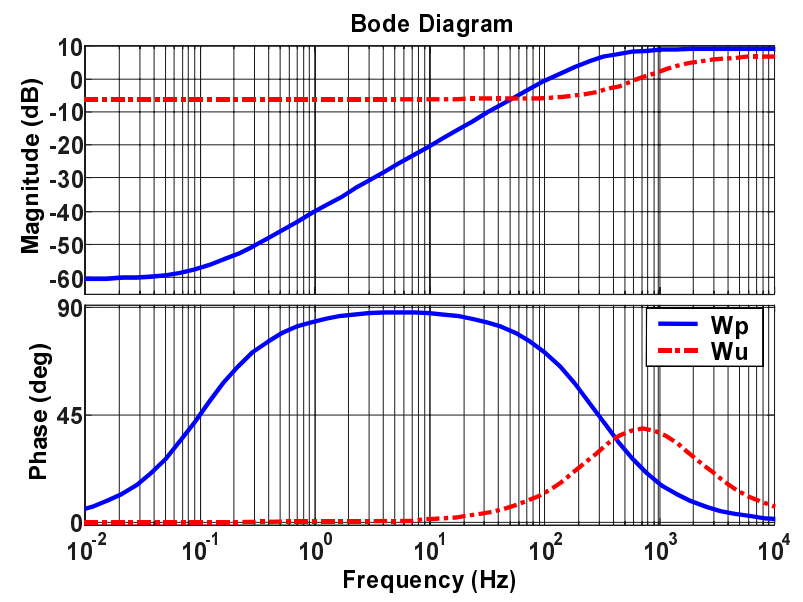

Fig. 6. Frequency response of $W_{p}$ and $W_{u}$ the design also checked the balanced reduction procedure. It was found that a $5^{\text {th }}$ order controller is effective. Figure 7 shows a comparison situations before and after reduction.

Figure 8 shows the SIMULINK blocks. The block plan shows the AFM tip-sample operated in the contact mode. The simulation included the effects of the computer implementation. The quantizer and saturation limits simulated the ADC; the saturation and Zero-Order-Hold $(\mathrm{ZOH})$ simulated the DAC device. The resolution of the $\mathrm{AD} / \mathrm{DA}$ card was 12 bits.

The simulation also included the uncertainties due to unmodeled dynamics and system nonlinearities. The tipsample interaction force was set to $20 \mathrm{nN}$. The sample variation was a sinusoidal profile with a $20 \mathrm{~nm}$ amplitude and $20 \mathrm{~Hz}$ frequency. Figure 9 (a) shows the reference profile, and Fig. 9(b) shows the control effort. The force between the tip and sample was kept at $20 \mathrm{nN}$ (Fig. 9(d)). Because the force error shown in Fig. 9(c) was very small, the control effort was sufficient accurately trace the sample profile. The variance of the force error relative to the setpoint was $-28 \mathrm{~dB}$.

The loop gain frequency response (GK) is shown in Fig. 10. One can see that the bandwidth was $200 \mathrm{~Hz}$.



Fig. 7. A bode diagram comparison of situations before and after order reduction

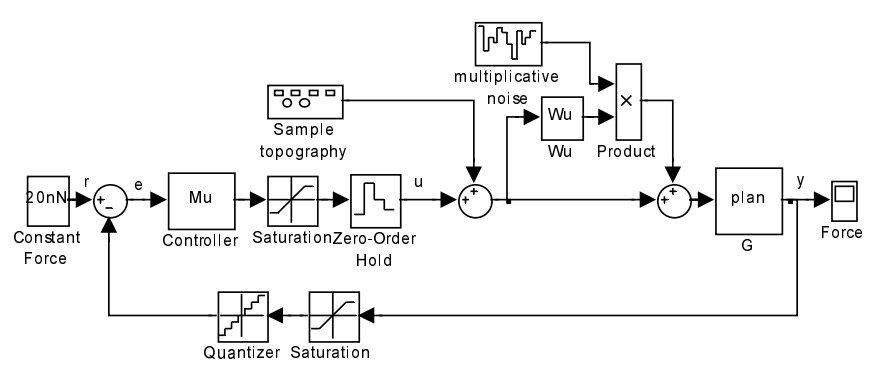

Fig. 8. Simulation block diagram of the closed-loop system 



Fig. 9. Simulation results for (a) the sample topography input;

(b) the response of the scanner; (c) the force error; and (d) the tip-sample interaction.

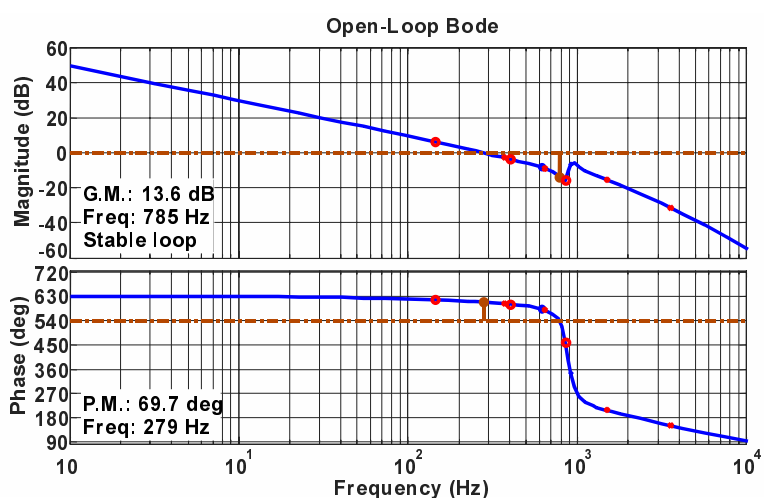

Fig. 10. The open-loop system frequency response

\section{EXPERIMENTAL RESULTS}

To demonstrate the performance of the controller, Fig. 11 shows the response when the tip was released. The range of $z$ motion was $6 \mu \mathrm{m}$. Before the tip was released, it was retracted (assuming a tip position of $3 \mu \mathrm{m}$ ). As shown in Fig. 11, the releasing action started at $20 \mathrm{~ms}$ and contacted the sample at $46 \mathrm{~ms}$. Due to the Van der Waals force, the tip-sample interaction due to an attractive force ranged from $20 \mathrm{~ms}$ to $46 \mathrm{~ms}$. The steady state error was $0.04 \mathrm{nN}$, which is smaller than 12-bit DAC resolution $(0.042 \mathrm{nN})$. The rise time of the controlled system was $4 \mathrm{~ms}$.
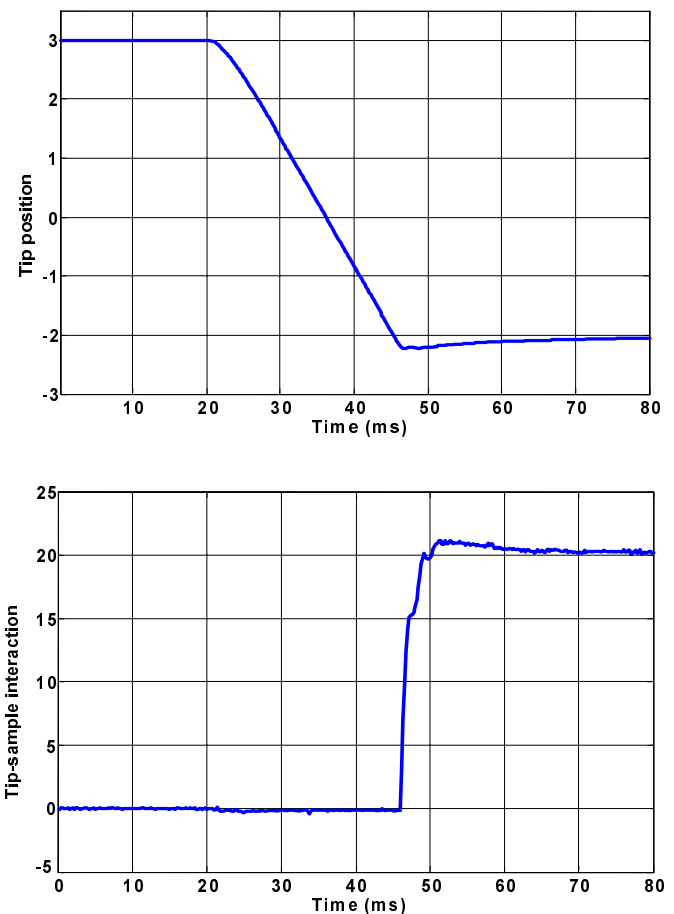

Fig. 11. The response when the tip was released. The force setpoint was $20 \mathrm{nN}$. 
The system performance test used a chirp signal to excite the set-point and collected the force error response. The error response plotted against the design criterion $W_{p}$ in Fig. 12 shows that the design is consistent.

Figure 13(a)-(d) shows images scanned at line scanning speeds ranging from under 10 to $80 \mathrm{~Hz}$. The Figs. 13(a) and (c) show the results obtained with the conventional PID control, and Figs. 13(b) and (d) show the results obtained with the proposed control. The sample was made from silicon nitride film deposited on a silicon substrate. The pattern on the sample was a $1 \mu \mathrm{m}$ square pattern with $2 \mu \mathrm{m}$ pitch. The height of the pattern was $75 \mathrm{~nm}$. It is clear that the proposed control obtained sharper images than the well-tuned PID controller, even at a very high scanning rate of $480 \mu \mathrm{m} / \mathrm{s}$ (comparing Figs. 13(c) and (d)). At such a high scan velocity, when we scanned a $3 \mu \mathrm{m} \times 3 \mu \mathrm{m}$ area with 64 pixels, the scan time was only $400 \mathrm{~ms}$.

The force error topography obtained at a $10 \mathrm{~Hz}$ linescan rate with the $\mu$-synthesis controller is shown in Fig. 14. The root mean square (RMS) of the force error under the proposed control was $0.154 \mathrm{nN}$, which is $-40 \mathrm{~dB}$ relative to the force set-point; therefore, the data shown in Fig. 13 are reliable with less than a $1 \%$ measurement error.

Comparing the error spectrum shown in Fig. 15 with the design specification $W_{p}$ again confirms the effectiveness of the control. Notice that the sampling frequency for the control was $10 \mathrm{KHz}$, and that scanning was performed at $10 \mathrm{~Hz}$. That left 250 data points with the smallest frequency point at around $20 \mathrm{~Hz}$. Comparing the images scanned at high scanning rates, one easily see that the micro pattern obtained with the proposed control is still distinguishable, while that obtained with the PID control shows only a small blip (Figs. 13(c) and (d)).

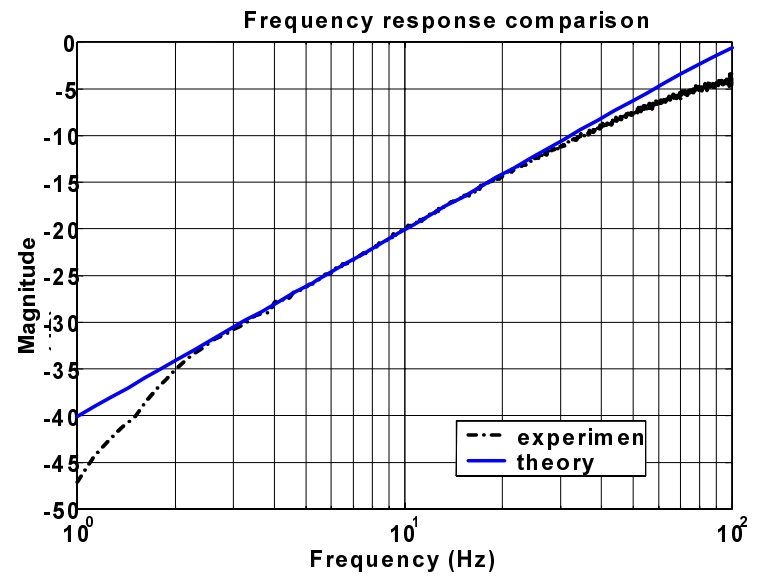

Fig. 12. Frequency response comparison. The solid line is the performance upper bound $\left(W_{p}\right)$ from setpoint to force error; the dashed line is the experimental frequency response from setpoint to force error.
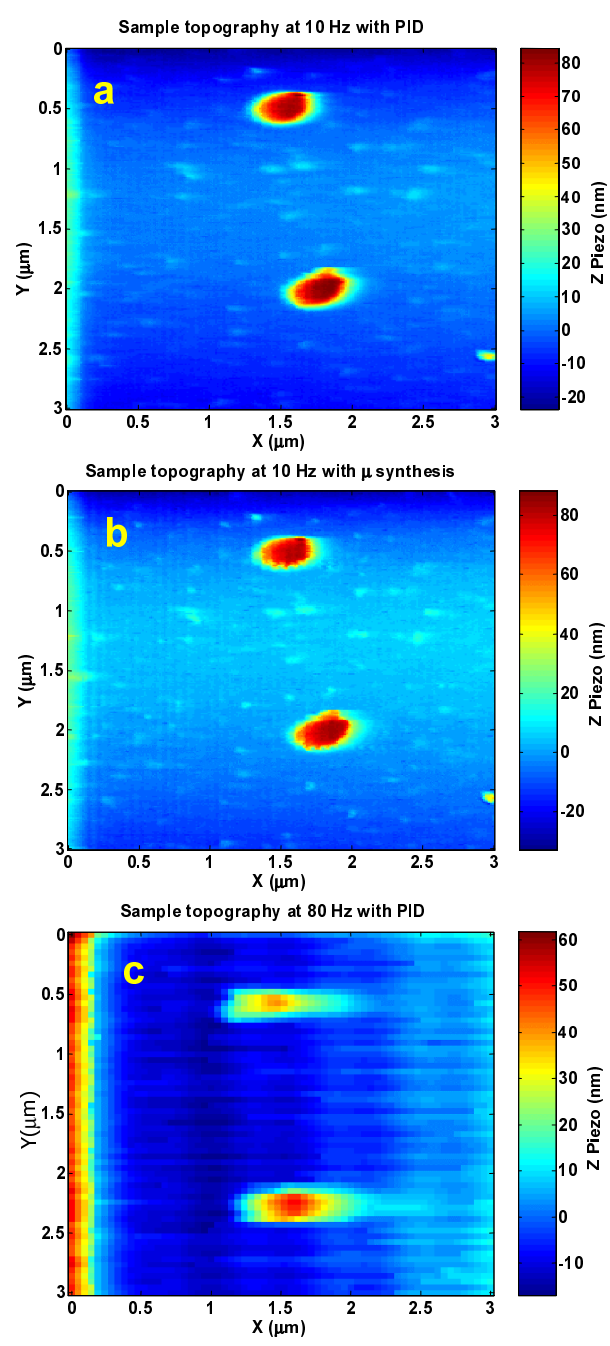

Sample topography at $80 \mathrm{~Hz}$ with $\mu$ synthesis



Fig. 13. The sample topography obtained at a $10 \mathrm{~Hz}$ line scan rate with the PID controller.

\section{SUMMARY AND CONCLUSIONS}

In this study, we modified a commercial AFM system and used it to test our proposed control method. A mathematical model was derived as the basis for the controller synthesis. Controllers were designed and implemented based on the $\mu$ control synthesis procedure. The $\mu$ 


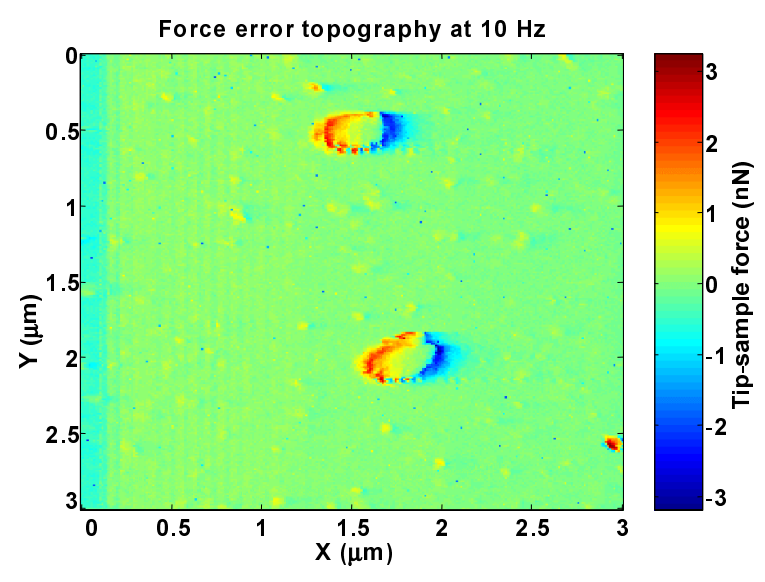

Fig. 14. The force error topography obtained at a $10 \mathrm{~Hz}$ line scan rate with the $\mu$-synthesis controller.

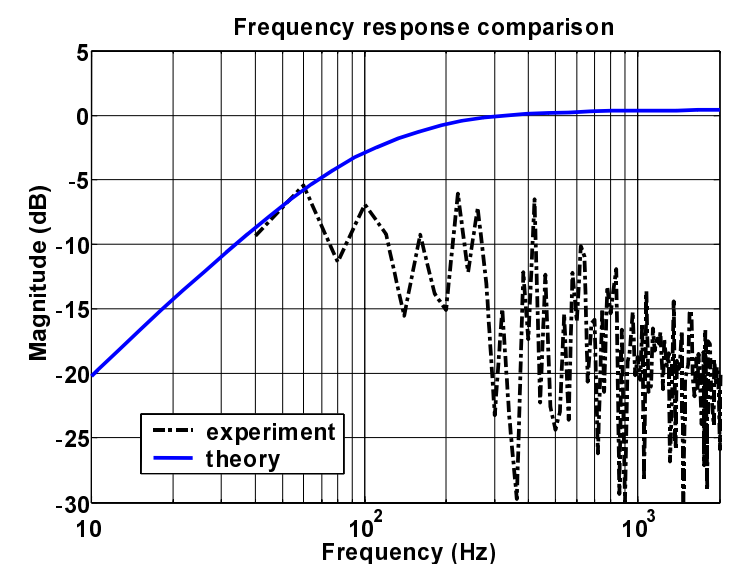

Fig. 15. Frequency response comparison. The solid line shows the performance upper bound $\left(W_{p}\right)$ from setpoint to force error; the dashed line shows linear spectrum of one line of force error data.

synthesis procedure considers the system nonlinearity and unmodeled dynamics with a model uncertainty. It is important to note that the proposed procedure also enables specification of the sample profile tracking error over the frequency domain. Therefore, it is possible to quantify the percentage of tracking error remaining in the measurement data. The resultant controller is able to achieve a bandwidth of $200 \mathrm{~Hz}$. Scanned images show that the proposed control achieves superior performance compared to the conventional control. Most important of all, the synthesis procedure allows one to access an error tolerance so that one can establish an error bound for the measurement profile.

\section{ACKNOWLEDGMENTS}

This project was supported in part by the Ministry of Education, R.O.C., and in part by the National Science Council, R.O.C., under project number NSC 91-2213-E002-028.

\section{REFERENCES}

1. "The National Technology Roadmap for Semiconductors, Technology Needs, 2001 Edition," Semiconductor Industry Association (2001).

2. Schitter, G., P. Menold, H.F. Knapp, F. Allgower, and A. Stemmer, "High Performance Feedback for Fast Scanning Atomic Force Microscopes," Rev. Sci. Instrum., Vol. 72, No. 8, pp. 3320-3327 (2001).

3. Battiston, F.M., M. Bammerlin, C. Loppacher, R. Luthi, E. Meyer, and H.-J. Guntherodt, "Fuzzy Controlled Feedback Applied to a Combined Scanning Tunneling and Force Microscope," Appl. Phys. Lett., Vol. 72, No. 1, pp. 25-27 (1998).

4. Fan, Y.C., M.G. Feemster, D.M. Dawson, and N. Jalili, "Nonlinear Control Techniques for the Atomic Force Microscope System," Proc., ASME Int. Mech. Eng. Congr. Exposi., New Orleans, Louisiana, pp. 1-8 (2002).

5. Osamah, M., EI Rifai, and Y.-T. Kamal, "Dynamics of Contact-Mode Atomic Force Microscopes," Proc. Amer. Contr. Conf., Chicago, Illinois, pp. 2118-2122 (2000).

6. Ashhab, M., M.V. Salapaka, M. Dahleh, and I. Mezic, "Control of Chaos in Atomic Force Microscopes," Proc. Amer. Contr. Conf., Albuquerque, New Mexico, pp. 196-202 (1997).

7. Osamah, M., EI Rifai, and Y.-T. Kamal, "In-Contact Dynamics of Atomic Force Microscopes," Proc. IEEE/ASME Int. Conf. Adv. Intell. Mechatron., Como, Italy, pp. 1325-1328 (2001).

8. Maugis, D., "Adhesion of Spheres: The JKR-DMT Transition Using a Dugdale Model,“ J. Colloid Interface Sci., Vol. 150, No. 1, pp. 243-269 (1992).

9. Balas, G.J., J.C. Doyle, K. Glover, A. Packard, and R. Smith, " $\mu$-Analysis and Synthesis Toolbox," MUSYN Inc. \& The Math Works, Inc. (2001).

10. Tymerski, R., "Worst Case Stability Analysis of Switching Regulators Using the Structured Singular Value," IEEE Trans. Power Electron., Vol. 11, No. 5, pp. 723-730 (1996).

11. Wallis, G.F. and R. Tymerski, "Generalized Approach for $\mu$ Synthesis of Robust Switching Regulators," IEEE Trans. Aerosp. Electron. Syst., Vol. 36, No. 2, pp. 422-430 (2000). 


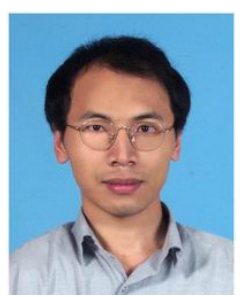

Kuo-Jung Lan received his B.S. degree from National Taiwan University in 2000. He is currently a doctoral candidate in the Department of Mechanical Engineering at NTU. His research interests include automatic control, active vibration isolation, and scanning probe microscope system. His theoretical research interests includes PID control, LQG control, continuous/discrete sliding mode control, $\mu$-Synthesis, and robust control.

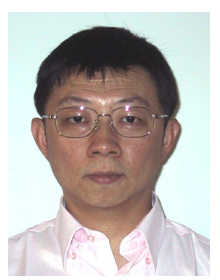

Jia-Yush Yen (M'87) became a member of IEEE in 1987. He was born in Taipei, Taiwan. He received the B.S. degree in mechanical engineering from National Tsinghua University, Hsinchu, Taiwan in 1980, and the M.S. degree from University of Minnesota, MN, in 1983. In 1989 he received the Ph.D. degree in mechanical engineering from University of California, Berkeley, CA. During his study at Berkeley, he received the IBM Graduate Fellowship in 1984-1985. Since 1989, he has been with National Taiwan University, Taipei, Taiwan, where he is currently a Professor of Mechanical Engineering. He served as the treasurer of the Control System Chapter in IEEE Taipei Section in 1992. He also served as consultant for many companies including Industrial Technology Research Institute, Taiwan and C Sun MFG. Ltd., Taiwan. His research interests are in the areas of modeling and control of electromechanical systems, especially in precision control of computer peripherals and precision measurement systems. He is also a member of the ASME.

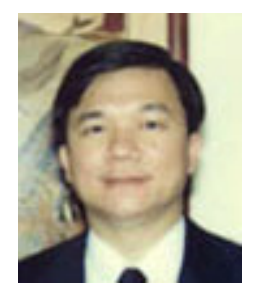

Chih-Kung Lee received his M.S. and Ph.D. degrees from Cornell University, in 1985 and 1987 respectively, with a major in theoretical and applied mechanics, and a minor in physics. Professor Lee is also concurrently the Director of Nondestructive Testing Laboratory at Tjing-Ling Industrial Research Institute, as well as an advisor to the Ministry of Education in charge of nanotechnology and flat panel display technology. Other duties include being the Director of the Planning Division of the Commission on Research and Development, National Taiwan University. His research interests are MEMS and nanotechnology system, automation technology, design \& construction of optoelectronic systems, optoelectronic and piezoelectric system, precision metrology, and biochip systems.

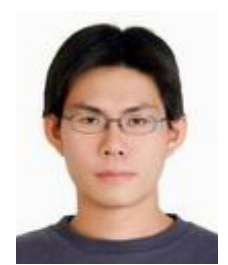

Yi-Ming Lin was born in Taipei, Taiwan on October 19, 1978. He received the B.S degree in 2001 and the M.S degree in 2003 in mechanical engineering from National Taiwan University.

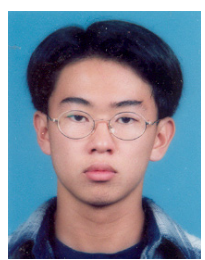

Cheng-Hung Chen is currently a doctoral candidate in the Department of Mechanical Engineering at NTU. 